\title{
Progression of Cell Wall Matrix Alterations during Aerenchyma Formation in Pisum sativum Root Cortical Cells
}

\author{
Timothy J. Pegg ${ }^{1}$, Richard E. Edelmann ${ }^{1}$, and Daniel K. Gladish ${ }^{1}$ \\ 1. Miami University, Department of Biology, Oxford, USA.
}

Predicted climatic changes necessitate investigation into crop resilience in low-lying farmlands threatened by prolonged periods of soil flooding due to increased rainfall [1]. Understanding plant adaptation mechanisms to prolonged immersion conditions provides options for genetic modification of existing crops to create cultivars better suited to areas susceptible to flooding [2]. An important advancement in understanding flooding adaptation would be to elucidate developmental mechanisms behind structural changes experienced by plant root systems during immersion.

In this study, Alaska Early Bush Pea (Pisum sativum) seedlings were utilized to study the formation of air channels, called aerenchyma, in roots during prolonged periods of immersion in water. Aerenchyma formation aides plant survival in flooded conditions by ensuring sufficient oxygen levels in immersed root tissues [3]. Aerenchyma formation occurs through programmed cell death (PCD) and entails the modification of chemical components in root tissue cells.

Pisum sativum parenchyma cells within the center of the root (i.e. vascular stele region) exhibit chemical modification of carbohydrates in the primary cell walls matrix, ultimately resulting in cell wall degradation prior to the formation of aerenchyma. Specifically, cortical cells adjacent to aerenchyma undergo alterations in pectic polysaccharides (i.e. pectin) through the removal of methyl groups via the process of de-methyl esterification [4]. Modifications of this nature are hypothesized to allow enzymatic hydrolysis between pectin residues via polygalacturonase and pectin lyase activity, followed by pectin removal from primary cell walls prior to aerenchyma formation. Understanding the mechanism of polysaccharide alteration may generate useful models for future crop improvement projects investigating events such as lateral root emergence and leaf senescence that are predicted to share similarities in cell wall remodeling processes.

Experimental design consisted of germination of eight groups of 20 pea seeds were grown in 2-liter beakers, containing sterile vermiculite and deionized water, at $25^{\circ} \mathrm{C}$ for 5 days in complete darkness. Four seedling groups were flooded with deionized water on day 5 to stimulate aerenchyma formation, with another four groups in unflooded beakers serving as controls. Tissues $1.5 \mathrm{~cm}$ to $3 \mathrm{~cm}$ basally from the root tip were excised from experimental and control groups at four timepoints post-flooding (12, 24, 36 , and 48 hours), embedded in agarose and sectioned at $100 \mu \mathrm{m}$ prior to immuno-labeling. Samples from control and experimental groups were stained with either CCRC-M1 or CCRC-M100 monoclonal primary antibodies targeting xylan residues or LM19, JIM7 or JIM5 antibodies targeting pectin residues with varying degrees of de-methyl esterification. Secondary labeling was done with Alexa Fluor® 647 conjugates and viewed with an Olympus FV500 confocal microscope to identify localization patterns near root aerenchyma.

Notable results include parenchyma cell walls adjacent to newly formed aerenchyma in flooded pea roots displayed enhanced fluorescence signal when treated with antibodies against completely de-methyl esterified pectin residues (Fig. 1A, LM19) and partially de-methyl esterified pectin (Fig. 1C, JIM5), but 
significantly weaker signal when treated with antibody targeting mostly methyl-esterified pectin residues (Fig. 1B, JIM7). Unflooded control treatments displayed enhanced signal in centralized groups of cells in the root stele when treated with LM19 (Fig. 1D) and JIM7 (Fig. 1E) antibodies, but no noticeable areas of signal increase when treated with JIM5.

The results of this study suggest that de-methyl esterification of pectin in the center of stele structures of flooded roots is necessary to prepare this region for enzymatic activity required to degrade cell walls and form cavities large enough to become aerenchyma. Interestingly, images of JIM7 antibody binding suggest that elevated areas of methyl-esterified pectin are present in the center of unflooded root steles (Fig. 1E) but disappear during prolonged periods of flooding (Fig. 1B). This result supports one hypothesis that methyl-esterified pectin residues are less susceptible to the enzyme degradation and must be de-methyl esterified prior to proper cavity formation.

\section{References:}

[1] Douglas, I., Food Security 1(2) (2009), p. 127.

[2] Tester, M. and P. Langridge, Science 327(5967) (2010), p. 818.

[3] Shimamura, S., et al., Annals of Botany 106(2) (2010), p. 277.

[4] Hyodo, H., et al., PLoS ONE 8(11) (2013), p. e78949.
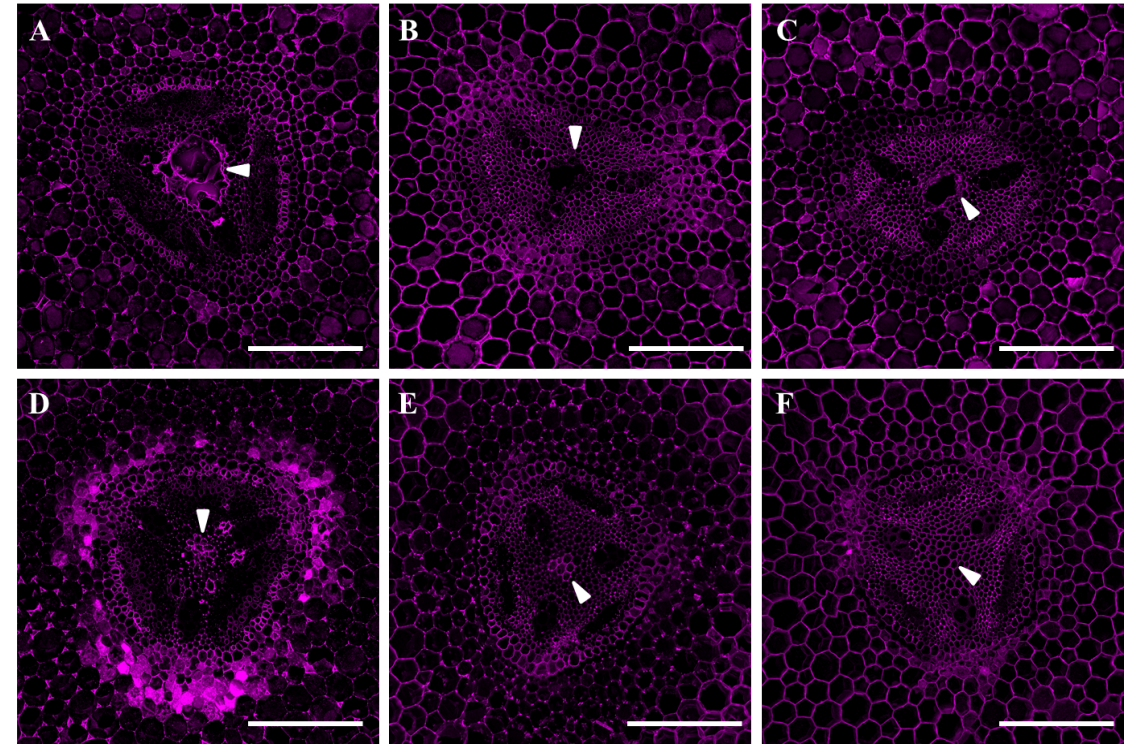

Figure 1. Monoclonal antibody binding patterns for (A-C) 48 hour flooded Pisum sativum root samples and (D-F) unflooded controls shown in magenta. (A, D) LM19, (B, E) JIM7 and (C, F) JIM5 antibody treatments. Center of root stele indicated by white wedges. Scale bars $=100 \mu \mathrm{m}$. 